\title{
Benign intracranial hypertension following vitamin A megadose
}

\author{
P J Perera, Y S Sandamal, S Randeny
}

Ceylon Medical Journal 2014; 59: 31

\section{Introduction}

Vitamin A megadose is given six monthly, starting from six months of age to all Sri Lankan children. Vitamin A is not excreted from the body, so excessive intake leads to toxicity. Within the months of July and August 2013, three infants were admitted to the Colombo North Teaching Hospital, Ragama, with evidence of benign intracranial hypertension (BIH) following vitamin A megadoses.

\section{Case report 1}

A six-month old boy from Ragama was admitted with vomiting and a bulging anterior fontanelle for three days. Symptoms started one day after receiving a vitamin A megadose. The child was on food containing carrots, but had no carotenaemia. He had no papilloedema. He had no vomiting after admission to hospital, and the fontanelle settled within 24 hours. Ultrasound scan (USS) of the brain was normal.

\section{Case report 2}

A seven-month old boy from Mahara, had vomiting and a bulging fontanelle one day after vitamin A megadose. The child was on food containing carrots and pumpkins. He had bilateral early papilloedema, but no other neurological signs. USS and CT of the brain were normal. Vomiting settled within 24 hours and the fontanelle became normal within three days.

\section{Case report 3}

A six-month old boy from Ja-ela was admitted with a bulging fontanelle one day after vitamin A megadose. The child was otherwise well. He had consumed papaya and carrots daily for the past two weeks before admission. On examination the child had carotenaemia. There was no papilloedema. The fontanelle became normal a day following admission. USS brain was normal.

All three children had a normal full blood count and CRP. All of them remained well when reviewed two weeks after discharge from hospital.

\section{Discussion}

$\mathrm{BIH}$ is diagnosed when intracranial pressure is raised without an underlying intracranial pathology. Headaches, vomiting and visual disturbances are common presenting symptoms of BIH [1]. In contrast to patients with intracranial mass lesions, consciousness and intellectual functioning remains normal in $\mathrm{BIH}$ [2]. Other than for papilloedema and occasional sixth cranial nerve palsy, neurological examination is often normal [3]. However, BIH can cause progressive deterioration of vision in about $10 \%$ of patients leading to blindness [4]. As in the three patients we describe, the widely open fontanelle buffers the effects of raised ICP, minimizing clinical features. However, as vitamin A supplementation is continued for up to five years of age, BIH occurring in a child with a closed fontanelle may result in serious visual complications.

In our patients imaging showed a normal brains and non-dilated ventricular systems which are prerequisites for diagnosing BIH [2]. Definitive evidence of BIH is a normal cerebrospinal fluid examination with elevated pressure. Lumbar puncture was not considered in our patients because they showed no evidence of central nervous system infections or pressure effects.

Breast milk is rich in vitamin A, and postpartum mothers receive 200,000 IU of vitamin A. Sri Lankan weaning foods contain carrots, green leafy vegetables, papaya and mangos, all rich in vitamin $\mathrm{A}$. About $25 \mathrm{~g}$ of carrots alone will provide an infant's daily requirement of vitamin A [5]. Selective administration of vitamin A, only to those children who need supplementation, may be a safer option considering the possible toxic effects of vitamin A megadoses.

\section{References}

1. Babikan P, Corbett J, Bell W. Idiopathic intracranial hypertension in children: the Iowa experience. Journal of Child Neurology 1994; 9: 144-9.

2. Solera D, Cox T, Bullock P, Calvered DM, Robinson RO. Diagnosis and management of benign intracranial hypertension. Archives of Diseases of Childhood 1998; 78: 89-94.

3 Gibby WA, Cohen MS, Goldberg HI, et al. Pseudotumourcerebri: CT findings and correlation with vision loss. American Journal of Roentgenology 1993; 160: 143-6.

4 Idiopathic Intracranial Hypertension. American Association for Pediatric Ophthalmology and Strabismus, 2012.

5 Park K. Text book of preventive and Social Medicine (19th Edition). Jabalpur: Banarsidas Bhanot 2009: 485-7.

Department of Paediatrics, Faculty of Medicine, University of Kelaniya, Ragama, Sri Lanka.

Correspondence: PJP, e-mail: <supipi1971@yahoo.com>. Received 12 September 2013 and revised version accepted 17 December 2013. Competing interests: none declared. 